RESEARCH ARTICLE

\title{
Meniscal Pain: US Guided Meniscal Wall Infiltration Versus Partial Meniscectomy, a Comparative Study
}

\author{
Clément Marion ${ }^{1 *}$, Marc Bouvard ${ }^{1}$, Alain Lippa ${ }^{2}$, Patrice Gardès ${ }^{2}$, François Lavallé ${ }^{2}$ and \\ Igor Benezis ${ }^{2}$
}

${ }^{1}$ Centre of Biology and Medicine of Sport de PAU, France

${ }^{2}$ Service for Orthopedic Surgery, France

*Corresponding author: Clément Marion, Center for Biology and Medicine of Sport de PAU, 64046 Pau cedex, France, E-mail: marionclement64@gmail.com

\begin{abstract}
Background: The increasing increase in the name of meniscal lesions leads to a reflection on their charge price. The aim of a treatment is to relieve the pain and to allow, as a priority, the smooth running of the daily and professional activities. The concept of meniscal economy is expanded by the medical community. We compared the benefit of infiltrations of the meniscal wall with the arhroscopic meniscectomy.

Material and method: We included 76 volunteer patients, suffering from meniscal pain, divided into 2 groups and coming from several centers. 41 consecutive patients in the infiltration group and simultaneously 35 consecutive in the arthroscopy/meniscectomy group. All patients completed an IKDC survey during the first visit. Another survey was carried out during a control visit at one year of treatment or via a telephone interview.

The primary endpoint was based on the evolution of IKDC score. The secondary endpoint was the resumption of initial physical activity, as well as its intensity level.

Results: There was no significant difference in the mean progression of the IKDC score between the infiltration and arthroscopy groups at one year of follow-up. $12.9 \pm 21.9$ vs. $19.8 \pm 18.2$. $P=0.142$. The rate of resumption of physical and occupational activity at one year is also not significantly different in the two groups. $P=0.797$. The rate of failure was very low in the 2 groups: $7.3 \%$ of the infiltrations against $2.8 \%$ of the arthroscopic surgeries.

Conclusion: The simplicity of the infiltration gesture, its low cost, the benign nature of its adverse effects, its low failure rate and the results obtained on this sample of 76 patients, encourage us to believe that the meniscal wall infiltration technique is Interesting to propose in $1^{\text {st }}$ intention to the patients suffering from meniscalgies without blocking episode.
\end{abstract}

\author{
Keywords \\ Knee, Meniscus, Meniscal pain, Meniscal wall infiltration, \\ Arthroscopic meniscectomy, Corticosteroids
}

\section{Introduction}

The increase in the practice of sport and physical activity in the general population, and the direct link that the people attributes to their health, lead to a steady increase [1] the number of knee injuries, including meniscal. The incidence of meniscal tears is $9 / 10000$ men and 4.2/10000 women. The sex ratio is $2 / 1$. Medial meniscus is injured in nearly $3 / 4$ of cases [1].

Meniscal injuries are active's men lesions. The young population is mostly affected, often by traumatic injuries. In a population of young athletes, Mitchel, et al. have counted between 2007 and 2013 about 5 injuries per 100000" athlete's expositions" [2]. However, the degenerative processes also cause meniscal tears among sportsmen of any age.

The goal of treatment is to relieve pain and allow priority, the smooth running of activities of daily life and work. Secondarily, it may be useful to a resumption of sports activities, without increasing the degenerative risk. Hundred and forty thousand meniscal lesions surgeries in France every year, and this is increasing [3]. So we understand the interest of a medical alternative when it's possible for a public health perspective.

The advent of Magnetic Resonance Imaging (MRI)

Citation: Marion C, Bouvard M, Lippa A, Gardès P, Lavallé F, et al. (2018) Meniscal Pain: US Guided Meniscal Wall Infiltration Versus Partial Meniscectomy, a Comparative Study. Int J Sports Exerc Med 4:086. doi.org/10.23937/2469-5718/1510086

Received: January 12, 2018; Accepted: April 16, 2018; Published: April 18, 2018

Copyright: (c) 2018 Marion C, et al. This is an open-access article distributed under the terms of the Creative Commons Attribution License, which permits unrestricted use, distribution, and reproduction in any medium, provided the original author and source are credited. 
Table 1: The population of the study is described.

\begin{tabular}{|l|l|l|l|}
\hline & $\mathbf{n}=\mathbf{7 6}$ & $\begin{array}{l}\text { Infiltration } \\
\text { Group }\end{array}$ & $\begin{array}{l}\text { Arthroscopy } \\
\text { Group }\end{array}$ \\
\hline Men & $56(73.7 \%)$ & $31(75.6 \%)$ & $25(71.4 \%)$ \\
Women & $20(26.3 \%)$ & $10(24.4 \%)$ & $10(28.6 \%)$ \\
\hline Age $^{* *}$ & $47.9[41.0-59.2]$ & $47.5[41.6-58.0]$ & $49.8[40.9-61.9]$ \\
\hline
\end{tabular}

*Qualitative variables are expressed as frequency (percentage); *Quantitative variables are expressed as median [Q1-Q3].

examination with better sensitivity and specificity, has a thinner viewing characteristics of meniscal lesions. The choice of therapeutic strategy is thus more suitable.

The concept of « meniscus economy » is now widely adopted by the medical community. It is in this context that since conservation, infiltration meniscal wall may be an alternative therapy for isolated lesions or not, in the absence of mechanical blockage. The High Authority for Health (HAS) recommends medical treatment (analgesics, anti-inflammatory, infiltration of corticosteroids or hyaluronic acid) in the first time as part of degenerative lesions. A marked improvement in symptoms being observed in 10-25\% of cases at 6 months [4]. In the absence of pinching the tibiofemoral spaced meniscal transfixing, lesions consistent with clinical symptomatology in medical treatment fails, can then benefit from the more partial meniscectomy can (Expert consensus).

The question we asked ourselves is: Is there an interest in patients suffering from meniscal pain to propose ultrasound-guided meniscal wall infiltration in alternative to arthroscopic surgery?

The comparative study presented below was conducted in several centers in the Pyrénées Atlantiques (France).

\section{Material and Method}

\section{Population studied}

The study population consisted of 76 patients, men and women (Table 1), active, aged 15 minimum (to exclude any pathology related to growth), recruited consecutively in parallel in several centers: The Center of Biology and Sports Medicine from Pau (CBMS), and orthopaedic departments of Pau Hospital Center, Clinic Marzet of Pau and the Bayonne Hospital Center. The inclusions were held from May 1, 2013 to December 14, 2014.

Patients were included if they had a pain in the stifle spaced. Namely: Finding a sore point of medial or lateral meniscus in a knee flexed at $90^{\circ}$, by doing flexion/extension, valgus/varus movement, or by varying the rotation of the tibia.

The lesion could be traumatic or degenerative. MRI was required and proposed if such was not the case. The presence of a knee effusion was not an exclusion criterion, but the status of the cartilage had to be known. Inclusion was proposed at the first consultation, patients were voluntary.
Exclusion criteria were a history of mechanical symptoms type fleeting or permanent blocking of the knee, the lesions of the central pivot, a history of meniscal surgery and the lack of iconographic evidence of meniscal lesion. The population of the study is described in (Table 1).

\section{Procedure of the study (chronology and sequence of inclusion)}

The course of the study was presented to patients during the first consultation and then they filled a functional assessment questionnaire knee: International Knee Documentation Comittee (IKDC) [5,6].

The IKDC Subjective Knee at 1 year was performed during a consultation or control in a telephone interview.

Patient characteristics and contact information were collected as medical history, physical activity, the date of the proposed treatment and the type of initial functional complaint.

Patients were included either by a sport's doctor or by an orthopedic surgeon. The "meniscal wall infiltration" group consisted of 46 consecutive patients recruited at the Center of biology and sports Medicine from Pau as well as in the orthopedic surgery department of Pau Hospital Center, by two different practitioners. The group "arthroscopic meniscectomy", consisting of 43 patients recruited consecutively and in parallel through the orthopedic departments of Pau Hospital Center, Clinic Marzet of Pau and Bayonne Hospital Center. 4 surgeons have conducted the recruitment.

There was no randomization, and it is an open study for feasibility reasons.

The primary endpoint was based on the evolution of the IKDC side score of 100 . The secondary endpoint was the recovery of the initial physical activity and its intensity level.

Ultrasound guided meniscal wall infiltration technique: The patient is lying, knee flexed to $90^{\circ}$. The hip is in abduction and the knee is placed resting on a foam backing as needed if the gesture relates to the medial meniscus. However, it is left in neutral if the action relates to the lateral meniscus (Figure 1).

In parallel, a table was prepared. Covered with a sterile field, the necessary equipment is placed there:

- A cover and sterile gel for the ultrasound probe

- Compresses impregnated with alcoholic Betadine

- The prefilled syringe cortivazol $3.75 \mathrm{mg} / 1.5 \mathrm{ml}$ or dipropionate + betamethasone sodium phosphate 7 $\mathrm{mg} / \mathrm{mL}$ and intramuscular needle $21 \mathrm{G} 40 \mathrm{~mm}$

\section{- A dry dressing.}

We use a single pair of steril gloves to make this ges- 


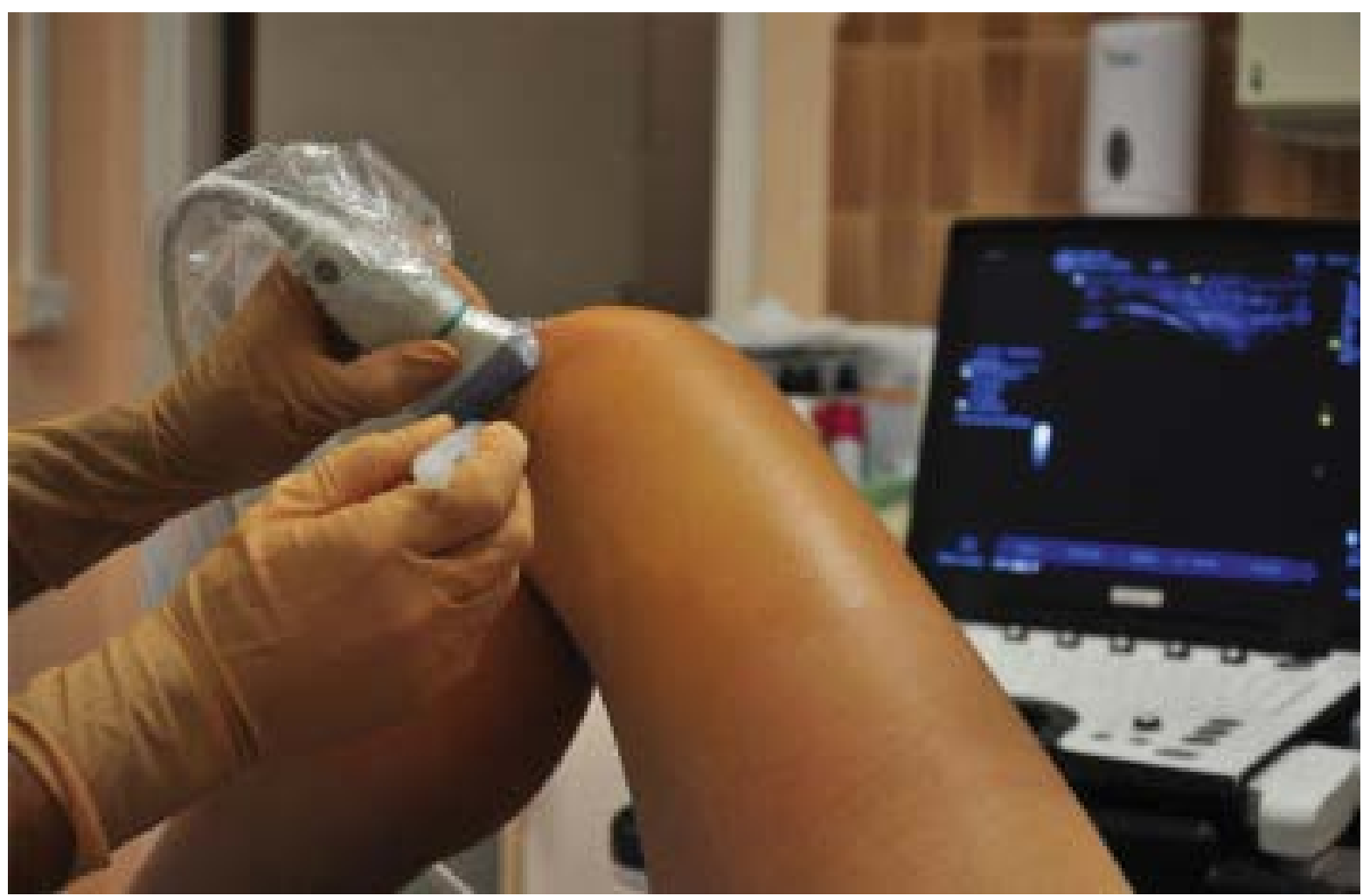

Figure 1: US guided infiltration technique.

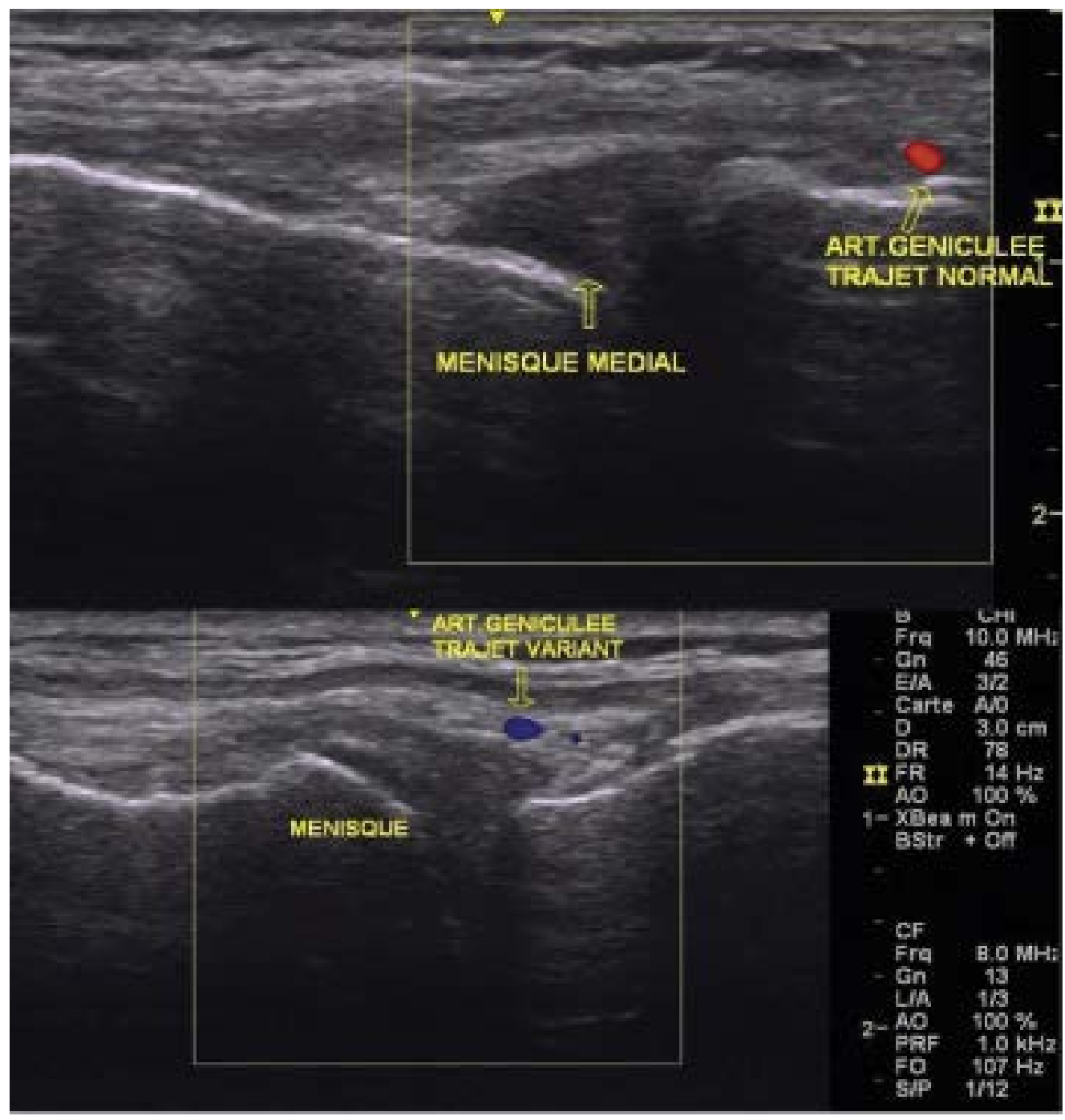

Figure 2: Variant way of genicular knee artery. Doppler window coronal ultrasonography. 
ture. After careful aseptic 3 times as recommended by the CLIN (Committee for the Fight against nosocomial infections), we perform an ultrasound location of meniscal lesion with or without a cyst. We take this moment to make a color Doppler windows for viewing the inferomedially or infero-side artery (Figure 2) of the knee that may have a varying distance along the line spacing [7]. This avoids an iatrogenic puncture thereof. This risk was highlighted for knee arthroscopy including [8].

It is important to shake the syringe before injection, the active ingredient are being suspended. It is not used for anesthesia. The puncture site is next to the joint line toward the meniscal lesion under ultrasound control. This will prevent accidental puncture of the artery or inferred-medial-lateral knee inferred. The needle is brought into contact with the meniscus. If it is too deep injection will not be possible with high resistance of the piston. The needle is then withdrawn slowly maintaining pressure on the plunger until a small resistance to the injection. The entire volume is injected near meniscus under the collateral ligament and not intra-articular. This may allow an injection into polylobed cysts.

We will carefully remove the needle under negative pressure so that cortivazol residues are not deposited in the skin tissue. This will avoid side effects such depigmentation, skin retraction.

The skin is then cleaned with alcohol and dried. A dry dressing is placed on the puncture site.

The patient needs partial rest for 8 days: Support and walking are authorized for the needs of everyday life.
If necessary, a work stoppage is proposed. The sporty rest is longer, about 15 days, accompanied by a gradual resumption of activities.

Patients with documented chondral lesions are offered a complementary injection of hyaluronic acid (HA) to D8.

Arthroscopic meniscectomy technique: Summary meniscectomy technique arthroscopic from EMC Surgical Technique [9].

This is an outpatient surgery or within a short hospital most of the time. The gesture is performed under spinal anesthesia, femoral block or general anesthesia.

The patient is supine with 2 variations according to the custom of the surgeon and the desired therapeutic range.

Using a tourniquet thigh provides better visibility intraarticular and its use has become routine. Conventionally, we are using a 2-way surgical approach. One for the instruments and one for the arthroscope.

The first time of the procedure is exploratory. After introduction of the arthroscope through the anterolateral and paddle through anteromedial, the operator observes the synovium, the state of cartilage covering surfaces, and feels the two sides of the menisci and cruciates ligaments.

Depending on the nature of meniscal lesions (which may be partly different from those diagnosed on MRI), is carried out meniscectomy gesture may be a single piece, by fragmentation or shaver.

Bucket handle lesions are not related to this work.

Table 2: The distribution of the type and intensity of physical activities are presented.

\begin{tabular}{|c|c|c|c|c|}
\hline & $n=76$ & Infiltration group & Arthroscopic group & $\mathbf{p}$ \\
\hline Men* & $56(73.7 \%)$ & $31(75.6 \%)$ & $25(71.4 \%)$ & \multirow{2}{*}{0.679} \\
\hline Women & $20(26.3 \%)$ & $10(24.4 \%)$ & $10(28.6 \%)$ & \\
\hline Overweight & $35(46.1 \%)$ & $17(41.5 \%)$ & $18(51.4 \%)$ & 0.385 \\
\hline \multicolumn{5}{|l|}{ Type of physical activity } \\
\hline \multicolumn{5}{|l|}{ Pivot } \\
\hline Ground impact & $16(21.1 \%)$ & $9(22.0 \%)$ & $7(20.0 \%)$ & \multirow[t]{3}{*}{0.274} \\
\hline \multirow[t]{2}{*}{ Landfill } & $19(25.0 \%)$ & $13(31.7 \%)$ & $6(17.1 \%)$ & \\
\hline & $41(53.9 \%)$ & $19(46.3 \%)$ & $22(62.9 \%)$ & \\
\hline Intensity & & & & \multirow{4}{*}{0.078} \\
\hline Low & $15(19.7 \%)$ & $5(12.2 \%)$ & $10(28.6 \%)$ & \\
\hline Medium & $35(46.0 \%)$ & $18(43.9 \%)$ & $17(48.6 \%)$ & \\
\hline High & $26(34.2 \%)$ & $18(43.9 \%)$ & $8(22.9 \%)$ & \\
\hline \multicolumn{5}{|l|}{ Sport recovery } \\
\hline No & $12(15.8 \%)$ & $7(17.0 \%)$ & $5(14.3 \%)$ & \multirow[t]{3}{*}{0.797} \\
\hline Yes & $42(55.3 \%)$ & $22(53.7 \%)$ & $20(57.1 \%)$ & \\
\hline Adaptation of type/intensity & $22(28.9 \%)$ & $12(29.3 \%)$ & $10(28.6 \%)$ & \\
\hline $\mathrm{Age}^{\star *}$ & $47.9[41.0-59.2]$ & $47.5[41.6-58.0]$ & $49.8[40.9-61.9]$ & 0.250 \\
\hline \multicolumn{5}{|l|}{ IKDC score } \\
\hline IKDC D365-IKDC D0 & $16.1 \pm 20.4$ & $12.9 \pm 21.9$ & $19.8 \pm 18.2$ & 0.142 \\
\hline
\end{tabular}

"Qualitative variables are expressed as frequency (percentage); " $Q$ Quantitative variables are expressed as median [Q1-Q3] or Mean \pm SD. 
Is completed by thorough washing of the articulation.

Walking is possible immediately without outside help.

Rehabilitation is not recommended according to the recommendations of the HAS (High Health Autority) [4]. It is important to inform patients that the consequences of a lateral meniscectomy are generally longer than a medial meniscectomy.

\section{Data collection}

The initial information was gathered during the first consultation with the answers to first IKDC survey. Responses to the second IKDC survey were collected at a control consultation, or in a telephone interview conducted by a single examiner.

\section{Statistics}

Data were entered into an Excel table with anonymisation. Statistical analysis was performed with SAS version 9.4 software. The descriptive analysis of the population was carried out with a FREQ procedure for categorical variables and the MEANS procedure for quantitative variables. The average, standard deviation, median and the $1^{\text {st }}$ and $3^{\text {rd }}$ quartiles were calculated for age and IKDC score. The existence of a relationship between qualitative variables uses Chi-2 tests. To study the relationship between a categorical variable and another quantitative (age and group; IKDC score and group), a Student t test was used. For the evolution of IKDC score, $t$ test was performed on paired variables. The significance threshold of statistical tests was set at $p<0.05$.

\section{Results}

\section{Population characteristics}

All the results are presented in (Table 2). We selected 41 patients in the infiltration group and 35 in the surgery group to analyze the results. There are 3 lost to only in the infiltration group.

The average age of patients was 47.9 years. $41.5 \%$ of patients in the infiltration group was overweight (BMI > 25 ) against $51.4 \%$ in the group arthroscopy.

$100 \%$ of patients said they were active. The distribution of the type and intensity of physical activities is presented in (Table 2). The intensities respectively correspond to $<1$ hour/week, between 1 and 4 hours, and $>4$ hours/week for low, medium, and strong.

The use of additional treatment of HA injection in connection with chondral lesions documented concerned $68 \%$ of patients in the infiltration group against only $25.7 \%$ of patients in arthroscopy group. Side effects were minimal, with only one case of injection pain for the infiltration group and one case of complex regional pain syndrome favorable to one year in the arthroscopy group.

\section{Analysis on the evaluation criteria}

Infiltration group: (Table 2) The primary endpoint was based on the evolution of the IKDC side score on 100. It was $12.9 \pm 21.9$ on average in the infiltration group (D365-D0). The minimum was -51.7 and maximum 57.5 .

3 patients were defeated by infiltration of treatment (persistent pain due clinically meniscus: see Part 2). They need a surgery (arthroscopic meniscectomy), respectively, 2, 7 and 12 months after meniscal wall infiltration. For the purposes of the study, their IKDC score at D365 was 0 .

In addition, one patient had a partial efficiency with a new infiltration motivated 9 months after the first. At one year of the beginning of the treatment, he considered himself in the same clinical condition before infiltration. To date, it has not been operated.

The secondary endpoint was the recovery of the initial physical activity and its intensity level. We can index in this group 7 recovery absences (one on the doctor's advice for Advanced chondropathy and another following an off-topic surgery) a year from the beginning of the treatment.

12 patients returned by adapting the type or intensity of activity: Reduction in the frequency or shift to landfill activities. In total, we count $3(7.3 \%)$ patients failing infiltration to a year of treatment.

Arthroscopic group: The primary endpoint was $19.8 \pm$ 18.2 on average in the arthroscopic group (IKDC D365-D0). The minimum was -9.2 and the maximum 60.9 .

Concerning the secondary endpoint, was able to list in this group 5 lack of recovery of physical activity. A patient for cervical problems, another following surgery on the contralateral knee, a third for an intervention of total hip replacement, a last for knee pain due to patellofemoral chondropathy. Note that the fifth patient was also forced to stop his professional activity (construction worker). 10 patients returned by adapting the type or intensity of activity: Reduction in the frequency or shift to landfill activities.

One patient $(2.8 \%)$ was considered failed surgery (no resumption of professional activity because of persistent knee pain).

\section{Comparative analysis}

The comparative analysis showed no statistically significant relationship between the treatment solution and various criteria: Sex, overweight, type of activity, intensity of practice, age, changes in the IKDC score and sport recovery. All $\mathrm{p}>0.05$.

The lack of statistically significant relationship between the descriptive variables of the population and the therapeutic solution, reassures us about the comparability of the two groups.

Regarding evaluation criteria, the analysis does not show statistical difference between the 2 groups. 


\section{Analysis of subgroups}

We chose to repeat the statistical analysis of the primary endpoint for several subpopulations.

Women: $(n=20)$, no reveal any statistically significant difference in the mean change in IKDC score between D0 and D365. The variation in the IKDC score was $15.8 \pm 27.7$ vs. $27.6 \pm 23.8$, respectively in the infiltration and arthroscopy groups. $p=0.323$.

Men: $(n=56)$, this difference was not significant. $p$ $=0.327$.

In overweight individuals from our sample $(n=35)$, the change in the IKDC score between D0 and D365 is not significant between the infiltration group and the arthroscopic group. $p=0.233$. We note that the mean change was $15.1 \pm 14.8$ vs. $22.3 \pm 19.6$, respectively in the infiltration and arthroscopy groups.

Finally, regarding the intense athletes ( $n=26$ ), we find no significant difference in the mean change in IKDC score. $p=0.357$.

\section{Discussion}

\section{Reminder of results and summary}

The absence of statistical relationship between the descriptive criteria of population and membership in one group or another therapeutic solution allows us to base our analysis on comparable groups. Indeed, all tests have returned to $p>0.05$ (see Table 2).

It was not possible to identify a standard population by analysis. Population distribution in the 2 treatment groups were homogeneous and symmetric.

We performed this study to show any difference between the therapeutic solution proposed to meniscal pain and evolution of functional IKDC score at 1 year. The lack of significance of statistical tests leads us to affirm that there is no statistical link on our sample of 76 patients, between the evolution of the score and the treatment group.

To allow analysis of all the data, IKDC scores at 1 year of the 3 patients considered in check in the infiltration group were counted as equal to 0 . Indeed, it was impossible to list a score that corresponds most precisely to reality among patients operated. They should have complete IKDC tests on memories, which we have excluded. The 0 score seems harsh but further strengthens the weight of no significant difference between the 2 treatment groups on the evolution of the IKDC score at 1 year.

For the distribution of types of physical activity, we selected 3 subcategories related to the stresses transmitted to the meniscus. The secondary endpoint was based on the recovery of physical activity. We felt it important to mention the recovery adaptation of a less binding activity to the meniscus. On this criterion, it was not found statistically significant differences between the 2 treatment groups.

Despite no significant difference on the criteria of primary and secondary judgment, we can not conclude as to the non-inferiority of treatment compared to another. Indeed, the protocol and methodology of the study are not adequate.

\section{Socio-economic arguments}

One of the arguments of the infiltration technique is its lower cost [10]. Indeed, this represents a total of about $55 €$ :

- 1 pre-filled syringe of cortivazol $=4.64 €$

- Ultrasound guided infiltration $=40.02 €($ CCAM cotation)

- Material for infiltration (compress/bandage/Polyvidone lode) $=$ about $10 €$

Conversely, arthroscopic meniscectomy is listed NFFC004 $=€ 191.43$. Added to this : The ambulatory surgery package and the costs of disposable equipment for surgery. The average total cost was estimated in 2012 to $1485 €[11]$. Is a cost in arthroscopy 27 times higher than that of the infiltration.

The issue of the cost of these two supported had already been discussed in a test of Van de graaf, et al. [12].

On the other hand, a major issue is the return to work after treatment. In our teams, achieving infiltration of the meniscus leads to a work stoppage on average 8 days. In light of the recommendations of the Primary Health Insurance Fund, it ranges from 10 to 45 days for arthroscopy according to the patient work of sedentary way or with heavy load port.

Considering the three days of regulatory failure and on the basis of the daily allowance of a person at minimum wage ( $€ 67.69$ gross), the cost to society of this work stoppage is $€ 338.45$ for infiltration against $€$ $2,842.98$ for arthroscopy. About 8.5 times higher for arthroscopic surgery.

\section{Compared to other studies}

In initial work from M. Lequesne in 1970 [13], injection was carried out painful point on the joint line palpation. The easy access and the significant improvement of the technical qualities offer now to make this gesture (since 2012 in our team) under ultrasound guidance.

The few international publication on the infiltration of the meniscal wall limits the comparison with results from other teams. However, Vermesan, et al. published in 2013 the results of a study comparing arthroscopic meniscectomy to intra-articular injection of corticosteroids as part of degenerative tear of meniscus [14]. On 120 patients ( 60 arthroscopy and 60 intra-articular injections of corticosteroids) changes in functional scores 
and pain was favorable. However, there was a significant difference on the interim evaluation at one month for arthroscopy. This difference became non-significant at one year. Furthermore, the study has put forward pejorative prognostic factors for progression of symptoms such as obesity or under the initial chondral edema. These symptoms may be due to the meniscus or chondral degenerative.

In a study of high level of evidence, Herrlin S.V. and his team [15], highlighted the non-superiority of a protocol involving arthroscopy and rehabilitation versus rehabilitation alone as part of degenerative lesions of the medial meniscus. The monitoring was carried out over 5 years. The study population consisted of 96 patients randomized into 2 groups of therapeutic solution. An average improvement of all monitoring scores (Knee injury Osteoarthritis Outcome Score, Lysholm Knee Scoring Scale and Visual Analogue Scale) could be demonstrated in the 2 groups without statistically significant difference $(p<0.0001)$. A third of patients in the rehabilitation group only, not improved, was operated with an efficiency on their quality of life. This study justified, in their middle-aged patient sample with the medial meniscus lesion on MRI confirmed the proposal of a well-conducted rehabilitation in first intention before turning to surgery.

In May 2013, J. N. Katz and colleagues published similar results but on knees combining meniscal tears and osteoarthritis [16].

Concerning degenerative meniscal lesions, Sihvonen, et al. compared in a bold test, surgery to sham surgery [17]. It was not revealed significant difference between arthroscopic meniscectomy groups and sham surgery (terms of arthroscopic surgery were merely mimed the operating room) for 1 year on Lysholm functional score, and pain. The occurrence of serious adverse events or the use of complementary surgery does not differ in the 2 groups.

Khan $\mathrm{M}$, et al. have made the synthesis of these studies showing in a meta-analysis [18] about 7 randomized controlled trials concerning the non profit arthroscopic surgery versus non-surgical treatments in middle-aged patients with or without moderate osteoarthritis.

Whatever the proposed support, the interest of the well pipe rehabilitation is regularly highlighted. Neogi DS and collaborators also showed the positive effects of physical therapy protocol [19] for patients with degenerative lesions of the medial meniscus, in particular on the occurrence of osteoarthritis. The latter was however strongly correlated with body mass index. We can also cite the case report of Stensrud, et al. which has the benefits of a 3-month rehabilitation program on degenerative meniscal lesions [20].

Other team Osteras, et al. which in a pilot study [21] with 17 patients showed a beneficial effect of rehabili- tation from the meniscectomy on evaluation scores of depression and anxiety at 3 months follow-up.

More recently, Blanke, et al. [22] suggest that the injection of platelet-rich plasma (PRP) would be beneficial to the healing of meniscal lesions on MRI (decrease the intensity of the MRI signal) as well as the pain, with a 6 months follow-up. The small size of this study $(n=10)$ deserves confirmation.

Finally, there were minimal side effects in the two groups. Now under study from Basques, et al. [23] can be observed up to $1.17 \%$ of serious adverse events after arthroscopic meniscectomy. Among these include septic arthritis, episode thromboembolism, or complex regional pain syndrome. For infiltration, it may appear a pain in the hours following the gesture. The risk of infection also exists. As well as the risk of skin atrophy by the accidental deposition of corticosteroid in the epidermis. This difference is taken into account for the therapeutic decision.

\section{Study limitations}

We are aware that this is a study of a modest level of evidence. First, lack of randomization is a major obstacle to the power of this study. We know the difficulty of constructing randomized studies in surgery. In this study, the surgical decision rested with the only surgeon. Moreover, we have not separated the different types of meniscal tears to refine comparative analysis. All this also puts a brake on the comparability of groups.

On the other hand, it was difficult to include the 3 patients lost in the analysis. Indeed, calculating the change in the IKDC score was impossible with a score missing at D365. The analysis was therefore not by intention to treat because of these 3 patients from the infiltration group.

Regarding the study protocol, including the collection of results at D365, we could not see all patients at D365. The IKDC score were filled in autonomy for some patients and via a telephone call to others. This could change in part to patient responses.

Furthermore, a regularly raised by the authors on the subject concerns the post-treatment rehabilitation. In our protocol, post-treatment rehabilitation is not framed and could greatly differ from one patient to another. Then we read $[15,16,19,20]$ that the benefits of this support could be significant and comparable to arthroscopy for certain meniscal lesions.

The proportion of lesions that have healed spontaneously is also impossible to demonstrate. We can not measure the share of profit would amount to infiltration.

We were stopped by the unusual rate of active population (100\%), which does not correspond to the finding in the general population [24]. A selection bias exists, related to the structure via which we conducted this study (Center for Biology and Pau Sports Medicine). 
Finally, the recovery time was not part of the data collection elements.

Meanwhile, the use of additional treatment of HA injection is different in the 2 groups. It was $68 \%$ vs. $25.7 \%$ respectively in the infiltration groups and arthroscopy. Patients with degenerative chondral was not excluded from the protocol, it is necessary to balance the benefit of $\mathrm{HA}$ on the painful symptoms. In the series of our team in 2008 [25], we showed $50 \%$ failure of the meniscal infiltration in case of absence of HA injection associated in patients with chondral lesions. From this work, the injection of $\mathrm{AH}$ is proposed systematically in case of lesion Association.

The concept of economy meniscal animates the medical community when it comes to handle meniscal lesions. "This concept is based on three attitudes: The more partial arthroscopic meniscectomy, abstaining from any gesture (all meniscal tear does not mean meniscectomy) and meniscal repair" [4].

An important point is that our study compares the infiltration of meniscal wall to arthroscopic meniscectomy only when the meniscal repair is now a priority for surgical teams. Compare the infiltration of meniscal wall to all arthroscopic treatment would be difficult from a methodological point of view, considering the variability of the type of injury.

Some teams now offer tests to assess the interest of Platelets Rich Plasma infiltration (PRP) in meniscal lesions in white-white area [26]. Still others attach to histology and highlight the proliferation of mesenchymal stem cells after meniscal tears in the synovial fluid which could be a contributing factor to spontaneous healing [27]. Ong, et al. propose a summary of advances in therapeutic research in this area [28]. Ruiz Iban team working on the growth factor proliferation of post meniscal lesion on animal model [29]. Using these new research areas could offer good therapeutic prospects.

Parallel, teams working on cell biology and in particular the repair by prosthetic elements covered with biological tissue which was proliferated on the surface. For now, the ratings are too few.

Vrancken, et al. conducted a literature review about the place of transplants (allografts, partial or full prosthesis) when the lesions are too advanced to allow for repair. Unfortunately, at present, too little testing high level of evidence allow the use of these techniques [30].

These new therapeutic will ultimately enhance our therapeutic arsenal against meniscal pain.

\section{Conclusion}

Our study tends to confirm our initial hypothesis. The ultrasound-guided meniscal wall infiltration of corticosteroid is an effective option to deal meniscal pain, without blocking.
It does not show a significant difference in the score IKDC mean change between infiltration and arthroscopy groups to a year of follow-up.

The recovery rates of physical activity and work in one year is not significantly different between the two groups.

The characteristics of the population in the two groups were comparable. We do not reveal differences regarding the mean change in IKDC score at one year specifically for women and in overweight patients.

The simple gesture of infiltration, low cost (27 times less than arthroscopy), the mild character of its adverse effects, low failure rate and results on this sample of 76 patients are encouraging us to think the infiltration technique is interesting to offer in $1^{\text {st }}$ intention to patients suffering from meniscal pain without blocking episode.

Each of care actors meniscal pain is driven by the concept of meniscal economy. Our thinking and our results are part of this approach.

It is important to bear in mind the role of the HA, particularly in the treatment of pain of mixed origin (chondropathy + meniscus) and propose it to patients.

To support this hypothesis, it should perform a non-inferiority, randomized and controlled. In the interest of public health, the period of recovery of physical activity and/or professional could be part of the judging criteria in addition to the pain assessment scores and functional scores.

\section{Conflict of Interrest}

The Authors declare that there are no conflicts of interest.

\section{References}

1. Verdonk R, Almqvist F (2005) Lésions traumatiques des ménisques du genouTraumatic lesions of the knee meniscus. EMC - Rhumatologie-Orthopédie 2: 592-613.

2. Mitchel J, Graham W, Best TM, Collins C, Currie D, et al. (2015) Epidemiology of meniscal injuries in US high school athletes between 2007 and 2013. Knee Surg Sports Traumatol Arthrosc 24: 1-8.

3. Pujol, Nicolas, Boisrenoult, Beaufils, Philippe (2013) Les lésions méniscales traumatiques. EMC - Appar Locomoteur.

4. HAS (2008) Prise en charge thérapeutique des lésions méniscales et des lésions isolées du ligament croisé antérieur du genou chez l'adulte.

5. Crawford K, Briggs KK, Rodkey WG, Steadman JR (2007) Reliability, validity, and responsiveness of the IKDC score for meniscus injuries of the knee. Arthroscopy 23: 839-844.

6. van de Graaf VA, Wolterbeek N, Scholtes VA, Mutsaerts EL, Poolman RW (2014) Reliability and Validity of the IKDC, KOOS, and WOMAC for Patients With Meniscal Injuries. Am J Sports Med 42: 1408-1416.

7. Bouvard M, Marion C, Parier J (2016) Injections for the treatment of meniscus pain: Usefulness, technique and contribution of ultrasonography. J Traumatol Sport 33: 114-118. 
8. Chen NC, Martin SD, Gill TJ (2007) Risk to the lateral geniculate artery during arthroscopic lateral meniscal suture passage. Arthroscopy 23: 642-646.

9. Panisset JC, Philippe N (2002) Méniscectomie sous arthroscopie. EMC - Tech Chir - Orthopédie 44.

10. Site de la Caisse Primaire d'Assurance Maladie - Classification Commune des Actes Médicaux [Internet]. Classification Commune des Actes Médicaux.

11. (2012) Chiffres de l'Agence Technique de I'Information sur l'Hospitalisation [Internet].

12. van de Graaf VA, de Gast A, Poolman RW (2013) Arthroscopic meniscectomy: does it make sense in patients older than 45?. Ned Tijdschr Geneeskd 157: 6865.

13. Lequesne M, Bensasson M, Kemmer C, Amouroux J (1970) Treatment of "meniscalgia" with juxta-meniscal infiltration. Presse Med 78: 1941.

14. Vermesan D, Prejbeanu R, Laitin S, Damian G, Deleanu B, et al. (2013) Arthroscopic debridement compared to intra-articular steroids in treating degenerative medial meniscal tears. Eur Rev Med Pharmacol Sci 17: 3192-3196.

15. Herrlin SV, Wange PO, Lapidus G, Hållander M, Werner S, et al. (2013) Is arthroscopic surgery beneficial in treating non-traumatic, degenerative medial meniscal tears? A five year follow-up. Knee Surg Sports Traumatol Arthrosc 21: 358-364.

16. Katz JN, Brophy RH, Chaisson CE, de Chaves L, Cole BJ, et al. (2013) Surgery versus physical therapy for a meniscal tear and osteoarthritis. N Engl J Med 368: 1675-1684.

17. Sihvonen $R$, Paavola $M$, Malmivaara $A$, Itälä $A$, Joukainen A, et al. (2013) Arthroscopic partial meniscectomy versus sham surgery for a degenerative meniscal tear. $\mathrm{N}$ Engl $\mathrm{J}$ Med 369: 2515-2524.

18. Khan M, Evaniew N, Bedi A, Ayeni OR, Bhandari M (2014) Arthroscopic surgery for degenerative tears of the meniscus: a systematic review and meta-analysis. CMAJ 186: 1057-1064.

19. Neogi DS, Kumar A, Rijal L, Yadav CS, Jaiman A, et al.
(2013) Role of nonoperative treatment in managing degenerative tears of the medial meniscus posterior root. J Orthop Traumatol Off J Ital Soc Orthop Traumatol 14: 193-199.

20. Stensrud S, Roos EM, Risberg MA (2012) A 12-week exercise therapy program in middle-aged patients with degenerative meniscus tears: a case series with 1-year follow-up. J Orthop Sports Phys Ther 42: 919-931.

21. Østerås $H$, Østerås B, Torstensen TA (2012) Medical exercise therapy, and not arthroscopic surgery, resulted in decreased depression and anxiety in patients with degenerative meniscus injury. J Bodyw Mov Ther 16: 456-463.

22. Blanke F, Vavken $P$, Haenle M, von Wehren L, Pagenstert G, et al. (2015) Percutaneous injections of Platelet rich plasma for treatment of intrasubstance meniscal lesions. Muscles Ligaments Tendons J 5: 162-166.

23. Basques BA, Gardner EC, Varthi AG, Fu MC, Bohl DD, et al. (2015) Risk factors for short-term adverse events and readmission after arthroscopic meniscectomy: does age matter? Am J Sports Med 43: 169-175.

24. (2016) Eurobarometer March 2010.

25. Bouvard M, Juret A (2008) Role of meniscal wall steroid injections for meniscal knee pain: 214 cases. J Traumatol Sport 25: 4-10.

26. Wei LC, Gao SG, Xu M, Jiang W, Tian J, et al. (2012) A novel hypothesis: the application of platelet-rich plasma can promote the clinical healing of white-white meniscal tears. Med Sci Monit 18: 47-50.

27. Matsukura Y, Muneta T, Tsuji K, Koga H, Sekiya I (2014) Mesenchymal stem cells in synovial fluid increase after meniscus injury. Clin Orthop Relat Res 472: 1357-1364.

28. Ong E, Chimutengwende-Gordon M, Khan W (2013) Stem cell therapy for knee ligament, articular cartilage and meniscal injuries. Curr Stem Cell Res Ther 8: 422-428.

29. Ruiz Ibán MÁ, Comellas Melero N, Martinez-Botas J, Ortiz A, Diaz Heredia J (2014) Growth factor expression after lesion creation in the avascular zone of the meniscus: a quantitative PCR study in rabbits. Arthroscopy 30: 1131-1138.

30. Vrancken AC, Buma P, van Tienen TG (2013) Synthetic meniscus replacement: a review. Int Orthop 37: 291-299. 